\title{
A Map of the Initiatives That Harmonize Patient Cohorts Across the World
}

\author{
Ángel Rodríguez-Laso ${ }^{1}$, Laura Alejandra Rico-Uribe ${ }^{2}$, Christine Kubiak ${ }^{3}$, \\ Josep Maria Haro ${ }^{4}$, Leocadio Rodríguez-Mañas ${ }^{1,5,6}$ and José Luis Ayuso ${ }^{2,7,8 *}$
}

${ }^{1}$ Thematic Area for Frailty and Healthy Ageing of the Network of Biomedical Research Centers (CIBERFES), Instituto de Salud Carlos III, Madrid, Spain, ${ }^{2}$ Centro de Investigación Biomédica en Red de Salud Mental, Instituto de Salud Carlos III, Madrid, Spain, ${ }^{3}$ European Clinical Research Infrastructure Network (ECRIN-ERIC), Paris, France, ${ }^{4}$ Parc Sanitari Sant Joan de Déu, Barcelona, Spain, ${ }^{5}$ Biomedical Research Foundation, Hospital Universitario de Getafe, Madrid, Spain, ${ }^{6}$ Geriatric Department, Hospital Universitario de Getafe, Madrid, Spain, ${ }^{7}$ Department of Psychiatry, Universidad Autónoma de Madrid, Madrid, Spain, ${ }^{8}$ Instituto de Investigación Sanitaria Princesa, Hospital Universitario de La Princesa, Madrid, Spain

Keywords: clinical cohort, patient cohort, harmonization, integration, repository

OPEN ACCESS

Edited by:

Laura Beane Freeman, National Cancer Institute (NCl), United States

Reviewed by:

Yutong Samuel Cai, University of Oxford, United Kingdom Milagros Ruiz,

University College London, United Kingdom

${ }^{*}$ Correspondence: José Luis Ayuso joseluis.ayuso@uam.es

Specialty section:

This article was submitted to Life-Course Epidemiology and Social Inequalities in Health,

a section of the journal Frontiers in Public Health

Received: 11 February 2021 Accepted: 14 June 2021 Published: 15 July 2021

Citation:

Rodríguez-Laso Á, Rico-Uribe LA, Kubiak C, Haro JM,

Rodríguez-Mañas $L$ and Ayuso $\mathrm{JL}$ (2021) A Map of the Initiatives That Harmonize Patient Cohorts Across the World. Front. Public Health 9:666844.

doi: 10.3389/fpubh.2021.666844

\section{INTRODUCTION}

Integration of cohort studies allows taking advantage of already collected information to increase the sample size to study uncommon exposures, rare diseases, less strong associations, or very restricted populations (personalized medicine). It also allows to carry out standardized analyses and avoid publication bias compared to the analysis of published data (1-5). Nevertheless, the growing energy spent in conducting cohort studies across the world in the last decades has not been paralleled by an effort to make them accessible to the scientific community and harmonize their data. This last limitation moved the European Commission to fund the SYNergies for Cohorts in Health: integrating the ROle of all Stakeholders (SYNCHROS) coordination and support action, endowed with almost $€ 2$ million $^{1}$ from 2019 to 2021 . It aims "to establish a sustainable European strategy for the development of the next generation of integrated cohorts, thereby contributing to an international strategic agenda for enhanced coordination of cohorts globally, in order to address the practical, ethical, legal, and methodological challenge of optimizing the exploitation of current and future cohort data, toward the development of stratified and personalized medicine as well as facilitating health policy."

In order to achieve its objectives, the first activity proposed in SYNCHROS was to map the population, patient, and clinical trial cohort integration landscape. That would allow the project to have a first look at the challenges and tried solutions adopted by different groups, and, more importantly, it would provide a list of principal investigators of these initiatives who could be contacted for the process of developing the common strategy. This study reports the result of the mapping of the initiatives that integrate patient cohorts. The mapping of population cohorts will be reported elsewhere. The aim of the study was to obtain a non-exhaustive, but representative, list of these initiatives carried out in recent times in the world. To our knowledge, there is no other repository of integration initiatives of patient cohorts. Although excellent single cohort repositories exist, like the Maelstrom catalog, repositories of initiatives that integrate several patient cohorts could not be found.

This mapping will provide researchers with a useful tool to find initiatives on their areas of interest with whom they can share or analyze harmonized data.

\footnotetext{
${ }^{1}$ https://cordis.europa.eu/project/id/825884/es.
} 


\section{METHODS}

The initiatives included in the mapping were obtained from three different sources:

1. Systematic searches, carried out in MEDLINE and the Maelstrom catalog. ${ }^{2}$

2. Suggestions of potential initiatives to be included in the mapping provided by partners of the SYNCHROS consortium.

3. References and links provided by the initiatives detected in the two previous sources.

The inclusion criteria were as follows:

a) initiatives that integrated patient, clinical, or disease cohorts;

b) individual patient meta-analysis and mega-analyses; and

c) at least one cohort included in the initiative having information about the sample at two or more points of time (at least two waves).

The exclusion criteria were as follows:

a) initiatives that only integrate population cohorts or clinical trials, without including patient cohorts;

b) initiatives published before the year 2000; and

c) initiatives that did not provide information in English.

\section{Database Searches}

\section{MEDLINE Search}

The process started with searches restricted to papers published in English from 2000 to 2019 using the terms selected by consensus among the SYNCHROS partners. Those terms which obtained fewer than 500 hits were retained, and the abstracts of the hits were reviewed to find new terms that were used in subsequent searches. In some cases, the term "cohort" was added to these searches to limit the number of hits.

The final search strategy used is given as follows:

(cohort OR "prospective study" OR "longitudinal study" OR "individual meta-analysis"[All Fields] OR "individual participant data meta-analysis" [All Fields] OR “individual patient data metaanalysis"[All Fields] OR "individual meta analysis"[All Fields] OR "individual participant data meta analysis"[All Fields] OR "individual patient data meta analysis"[All Fields] OR "meta analysis using individual"[All Fields] OR "meta-analysis using individual"[All Fields] OR "meta analysis of individual"[All Fields] OR "meta-analysis of individual"[All Fields] OR "megaanalysis"[All Fields] OR "mega analysis"[All Fields])

\section{AND}

("harmonization study" OR “integration study" OR "integration initiative" OR "integrated study" OR "merged cohort" OR "data pooling" OR "pooled sample" OR "combined data" OR "combining data" OR "harmonized data" OR "harmonised data" OR "harmonizing data" OR "data harmonization" OR "data harmonisation" OR "data sharing" OR "common database" OR "multiple cohorts" OR "multiple longitudinal studies" OR "international consortium” OR "collaborative effort").

${ }^{2}$ https://www.maelstrom-research.org/maelstrom-catalogue.

\author{
AND \\ (“2000/01/01”[Date - Publication]: “2019/07/31”[Date \\ - Publication]) \\ AND \\ English[Language] \\ AND \\ Humans[MeSH]
}

\section{Maelstrom Catalog}

The Maelstrom research catalog, supported by the Research Institute of the McGill University Health Centre, "contains comprehensive information about epidemiological research networks and studies, and the data they have collected. It also provides information about harmonized data generated by these research networks."

We looked for initiatives included in the "Networks" section of the catalog.

\section{Selection of Initiatives}

Initiatives that were obtained from the systematic searches and provided by the partners were evaluated against the inclusion and exclusion criteria by two different investigators. In case of a disagreement, a third reviewer was consulted.

\section{Extraction of Information}

The following information was extracted from each initiative: name of the initiative, principal investigator, partners, name of the institution responsible for the initiative, funding resources, contact person, information source, whether the research team is currently active, main objectives, criteria for the cohorts to be included in the initiative, type of harmonization (prospective/retrospective), number of cohorts included in the initiative (the total number and the number of harmonized cohorts), whether more cohorts are foreseen to be harmonized, number of participants (the total number and the number of participants with harmonized data), age range of the sample, threats to representativeness of the sample, maximum number of variables that have been harmonized, including those where harmonization was not possible for all the cohorts, setting of the harmonized cohorts (local-regional/national/international, including country of origin of the cohorts), and a brief description of the population considered by the initiative.

All this information was retrieved from the webpage and/or the scientific article that presented the initiative. Missing information was requested from the principal investigators of the projects, who were contacted initially by email and, if there was no answer, by phone call or by post.

\section{ANALYSIS}

Results of the identification process of the initiatives are presented in Figure 1.

Partners of the SYNCHROS project provided 39 initiatives. Of those, 28 were excluded, mainly because there was no data harmonization or because eligibility could not be ascertained due to unresponsiveness from the principal investigators. The 


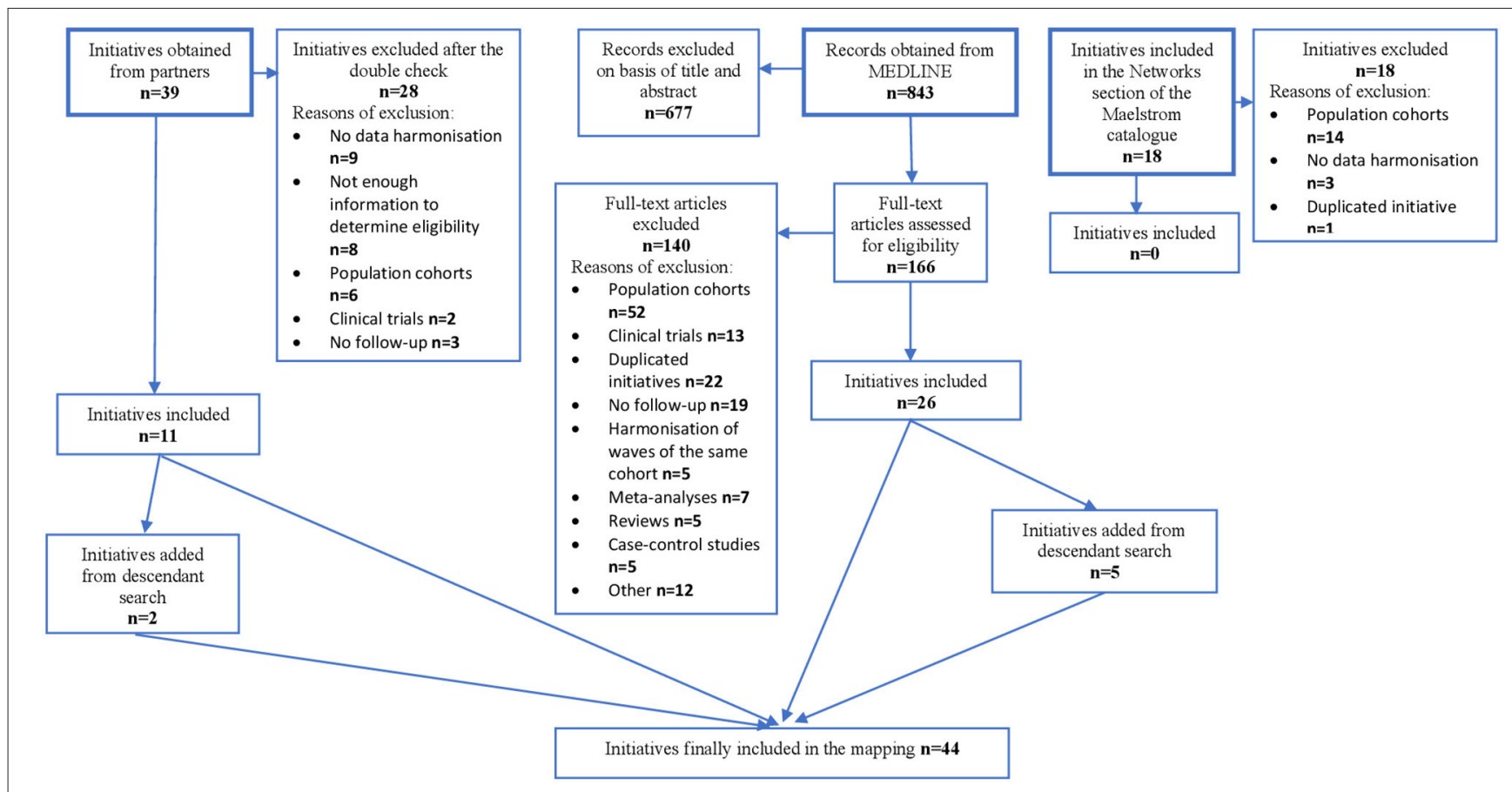

FIGURE 1 | Results of the search for harmonization initiatives of patient cohorts.

remaining 11 initiatives were selected. The descendent search from these initiatives provided two additional ones.

In the MEDLINE search, out of 843 hits obtained, 677 were excluded after reading their title and abstract. Of the remaining articles, 166 were read and, from those, 140 excluded. The main reasons for exclusion were that initiatives dealt only with population cohorts, that they had already been submitted by partners or already presented in another reference, or that the integration was only cross-sectional. In the end, 26 initiatives were selected. The reference list of these initiatives included five additional ones.

The search in the Maelstrom catalog only provided initiatives that harmonized population cohorts.

Overall, 44 initiatives were retrieved. They are presented in Table 1.

Table 1 shows a selection of the most relevant information obtained from each of the initiatives. Complete information can be found in the repository of the SYNCHROS project. ${ }^{3}$ They are ordered by types of diseases covered (starting with those which consider several diseases) and by alphabetical order. Of the 44 initiatives found, no further information could be obtained from principal investigators in almost half (20) of them.

Eight initiatives (BIOMAP, CINECA, EHDEN, ESCAPNET, HarmonicSS, HARMONY, Lifebrain, and ReCoDID) have recently started adding cohorts; 21 are led by active research teams; and 12 are adding, or considering adding, cohorts now. Nevertheless, there is plenty of missing information on the activity status of the initiatives.

${ }^{3}$ https://www.synchros.es.
In the selected initiatives, the most represented group of diseases is cancer (10 initiatives), followed by infectious diseases (8 initiatives, of which 5 focus on HIV) and cardiovascular disease (4 initiatives). There are five initiatives that have harmonized data from more than one type of disease. Other diseases and conditions producing a high burden in the high-income countries (6) are represented (dementia, osteoarthritis), but others included in this list (unipolar depressive disorders, alcohol use disorders, hearing loss, chronic obstructive pulmonary disease, diabetes mellitus, road traffic accidents) or poor-defined conditions with a well-defined impact on life-expectancy and quality of life (like back pain or functional deterioration) are missing. There is one initiative about a specific rare disease (Sjögren syndrome).

There is a sizable number of initiatives that have harmonized other types of cohorts in addition to patient cohorts. After Breast Cancer Pooling Project, BIOMAP, CLL-IPI, HARMONY, and the initiatives on obsessive-compulsive disorder and pulmonary embolism have harmonized at least one clinical trial cohort. CINECA, ESCAPE-NET, Lifebrain, and the project "Seasonal plasticity of cognition" have also harmonized population cohorts. BiomarCaRE and the National Cancer Institute Cohort Consortium have harmonized the three types of cohorts: patient, population and clinical trials cohorts.

Most of them (33) have an international scope, compared to seven national initiatives and one regional/local initiative. Two initiatives report that they include cohorts from across the world and eight initiatives incorporate cohorts from high- and low- and middle-income countries (LMIC); 30 (75\%) initiatives 
TABLE 1 | Initiatives that harmonize patient cohorts ordered by different categories of diseases (selected information).

\begin{tabular}{|c|c|c|c|c|c|c|c|c|c|}
\hline Initiative & Types of cohorts & $\begin{array}{l}\text { Region, Country } \\
\text { were the cohorts } \\
\text { were collected }\end{array}$ & Main objective & $\begin{array}{l}\text { Number of } \\
\text { cohorts with } \\
\text { harmonized data }\end{array}$ & $\begin{array}{l}\text { More } \\
\text { cohorts } \\
\text { foreseen to } \\
\text { be } \\
\text { harmonized? }\end{array}$ & $\begin{array}{l}\text { Number of } \\
\text { participants } \\
\text { with } \\
\text { harmonized } \\
\text { data }\end{array}$ & $\begin{array}{l}\text { Age range } \\
\text { of the } \\
\text { sample }\end{array}$ & $\begin{array}{l}\text { No. of } \\
\text { harmonized } \\
\text { variables } \\
\text { (Maximum) }\end{array}$ & Population \\
\hline $\begin{array}{l}\text { CINECA: } \\
\text { Common } \\
\text { Infrastructure for } \\
\text { National Cohorts } \\
\text { in Europe, Canada } \\
\text { and Africa }\end{array}$ & $\begin{array}{l}\text { Disease cohorts. } \\
\text { Population } \\
\text { cohorts }\end{array}$ & $\begin{array}{l}\text { Africa, Canada } \\
\text { and Europe }\end{array}$ & $\begin{array}{l}\text { To develop a federated cloud enabled } \\
\text { infrastructure to make population scale } \\
\text { genomic and biomolecular data } \\
\text { accessible across international borders, } \\
\text { to accelerate research, and improve the } \\
\text { health of individuals across continents }\end{array}$ & In progress & Possibly & In progress & $\begin{array}{l}\text { Birth to old } \\
\text { age }\end{array}$ & In progress & $\begin{array}{l}\text { The dataset provides a } \\
\text { diverse representation } \\
\text { of studies in rare } \\
\text { disease, common } \\
\text { disease and national } \\
\text { cohorts over time } \\
\text { (longitudinal) }\end{array}$ \\
\hline $\begin{array}{l}\text { CNODES: the } \\
\text { Canadian Network } \\
\text { for Observational } \\
\text { Drug Effect } \\
\text { Studies }\end{array}$ & Disease cohorts & $\begin{array}{l}\text { Canada, US and } \\
\text { UK }\end{array}$ & $\begin{array}{l}\text { Use collaborative, population-based } \\
\text { approaches to obtain rapid answers to } \\
\text { questions about drug safety and } \\
\text { effectiveness }\end{array}$ & $\begin{array}{l}\text { Depends on the } \\
\text { research question }\end{array}$ & No & $\begin{array}{l}\text { Depends on } \\
\text { the research } \\
\text { question }\end{array}$ & All ages & $\begin{array}{l}\text { Depends on } \\
\text { the research } \\
\text { question }\end{array}$ & $\begin{array}{l}\text { Population of Canada, } \\
\text { UK and US which is } \\
\text { prescribed or } \\
\text { dispensed drugs }\end{array}$ \\
\hline $\begin{array}{l}\text { EHDEN: European } \\
\text { Health Data and } \\
\text { Evidence Network }\end{array}$ & Disease cohorts & $\begin{array}{l}\text { All Horizon } 2020 \\
\text { member states } \\
\text { and associated } \\
\text { countries }\end{array}$ & $\begin{array}{l}\text { Harmonize in excess of } 100 \text { million } \\
\text { anonymized health records to the } \\
\text { OMOP common data model, supported } \\
\text { by an ecosystem of certified SMEs, and } \\
\text { technical architecture for a federated } \\
\text { network }\end{array}$ & & In progress & & 18 & Considerable & $\begin{array}{l}\text { European patients } \\
\text { aged } 18+\end{array}$ \\
\hline $\begin{array}{l}\text { MIRACUM: } \\
\text { Medical } \\
\text { Informatics in } \\
\text { Research and } \\
\text { Care in University } \\
\text { Medicine }\end{array}$ & Disease cohorts & $\begin{array}{l}\text { Seven states of } \\
\text { Germany }\end{array}$ & $\begin{array}{l}\text { The spotlight is here on the data } \\
\text { integration centers that will be } \\
\text { embedded in the hospital } \\
\text { IT-infrastructure and will facilitate the } \\
\text { collection and exchange of data within } \\
\text { the consortia university hospitals. } \\
\text { Furthermore, we will elaborate a } \\
\text { programme for strengthening medical } \\
\text { informatics by extending the academic } \\
\text { offer, including new professorships in the } \\
\text { field of medical informatics, a novel, } \\
\text { innovative master programme and } \\
\text { personnel training. The MIRACUM } \\
\text { partners have agreed to share data, } \\
\text { based on interoperable data integration } \\
\text { centers, develop common and } \\
\text { interoperable tools and services, realize } \\
\text { the power of such data and tools in } \\
\text { innovative IT solutions, which shall } \\
\text { enhance patient-centered collaborative } \\
\text { research as well as clinical care } \\
\text { processes, and finally to strengthen } \\
\text { biomedical informatics in research, } \\
\text { teaching and continued education }\end{array}$ & 11 & $\begin{array}{l}\text { No information } \\
\text { obtained }\end{array}$ & $\begin{array}{l}\text { No information } \\
\text { obtained }\end{array}$ & $\begin{array}{l}0 \text { to the } \\
\text { highest age } \\
\text { of patients }\end{array}$ & $\begin{array}{l}\text { No } \\
\text { information } \\
\text { obtained }\end{array}$ & $\begin{array}{l}\text { Patients attended in } \\
\text { hospitals of seven } \\
\text { German states }\end{array}$ \\
\hline
\end{tabular}


TABLE 1 | Continued

\begin{tabular}{|c|c|c|c|c|c|c|c|c|c|}
\hline Initiative & Types of cohorts & $\begin{array}{l}\text { Region, Country } \\
\text { were the cohorts } \\
\text { were collected }\end{array}$ & Main objective & $\begin{array}{l}\text { Number of } \\
\text { cohorts with } \\
\text { harmonized data }\end{array}$ & $\begin{array}{l}\text { More } \\
\text { cohorts } \\
\text { foreseen to } \\
\text { be } \\
\text { harmonized? }\end{array}$ & $\begin{array}{l}\text { Number of } \\
\text { participants } \\
\text { with } \\
\text { harmonized } \\
\text { data }\end{array}$ & $\begin{array}{l}\text { Age range } \\
\text { of the } \\
\text { sample }\end{array}$ & $\begin{array}{l}\text { No. of } \\
\text { harmonized } \\
\text { variables } \\
\text { (Maximum) }\end{array}$ & Population \\
\hline Sentinel initiative & Disease cohorts & US & $\begin{array}{l}\text { Serve as a system to analyze and } \\
\text { assess safety risks in FDA-approved } \\
\text { drugs and medical products using } \\
\text { electronic health data }\end{array}$ & 17 & $\begin{array}{l}\text { No information } \\
\text { obtained }\end{array}$ & 310 million & All ages & $\begin{array}{l}\text { No } \\
\text { information } \\
\text { obtained }\end{array}$ & $\begin{array}{l}\text { Population of US which } \\
\text { is prescribed or } \\
\text { dispensed drugs }\end{array}$ \\
\hline $\begin{array}{l}\text { BiomarCaRE: } \\
\text { Biomarker for } \\
\text { Cardiovascular } \\
\text { Risk Assessment } \\
\text { across Europe }\end{array}$ & $\begin{array}{l}\text { Disease cohorts. } \\
\text { Population } \\
\text { cohorts. Clinical } \\
\text { trials }\end{array}$ & $\begin{array}{l}\text { Australia, Europe, } \\
\text { Israel, Latin } \\
\text { America, New } \\
\text { Zealand, South } \\
\text { Africa, } \\
\text { United States }\end{array}$ & $\begin{array}{l}\text { Assess the value of established and } \\
\text { emerging biomarkers for cardiovascular } \\
\text { risk prediction }\end{array}$ & 4 & $\begin{array}{l}\text { No information } \\
\text { obtained }\end{array}$ & 8.746 & $\begin{array}{l}\text { No } \\
\text { information } \\
\text { obtained }\end{array}$ & $\begin{array}{l}\text { No } \\
\text { information } \\
\text { obtained }\end{array}$ & $\begin{array}{l}\text { Patients with coronary } \\
\text { heart disease or at risk } \\
\text { of developing it }\end{array}$ \\
\hline $\begin{array}{l}\text { CADISP: Cervical } \\
\text { Artery Dissection } \\
\text { and Ischemic } \\
\text { Patients }\end{array}$ & Disease cohorts & $\begin{array}{l}\text { Western Europe } \\
\text { and Turkey }\end{array}$ & $\begin{array}{l}\text { International Consortium performing } \\
\text { research on ischemic stroke in young } \\
\text { and middle-aged adults and in particular } \\
\text { on cervical artery dissection }\end{array}$ & $\begin{array}{l}\text { No information } \\
\text { obtained }\end{array}$ & $\begin{array}{l}\text { No information } \\
\text { obtained }\end{array}$ & $\begin{array}{l}\text { No information } \\
\text { obtained }\end{array}$ & $\begin{array}{l}\text { No } \\
\text { information } \\
\text { obtained }\end{array}$ & $\begin{array}{l}\text { No } \\
\text { information } \\
\text { obtained }\end{array}$ & $\begin{array}{l}\text { Cervical artery } \\
\text { dissection and } \\
\text { ischemic stroke } \\
\text { patients from some } \\
\text { Western European } \\
\text { countries and Turkey }\end{array}$ \\
\hline $\begin{array}{l}\text { Development and } \\
\text { validation of the } \\
\text { AMPREDICT } \\
\text { model }\end{array}$ & Disease cohorts & US & $\begin{array}{l}\text { The objective of this study was the } \\
\text { development of AMPREDICT-Mobility, a } \\
\text { tool to predict the probability of } \\
\text { independence in either basic or } \\
\text { advanced mobility } 1 \text { year after } \\
\text { dysvascular major lower extremity } \\
\text { amputation }\end{array}$ & 2 & $\begin{array}{l}\text { No information } \\
\text { obtained }\end{array}$ & 200 & $\begin{array}{l}\text { No } \\
\text { information } \\
\text { obtained }\end{array}$ & 38 & $\begin{array}{l}\text { Individuals undergoing } \\
\text { their first major lower } \\
\text { extremity amputation } \\
\text { because of } \\
\text { complications of } \\
\text { peripheral artery } \\
\text { disease or diabetes }\end{array}$ \\
\hline $\begin{array}{l}\text { ESCAPE-NET: } \\
\text { European Sudden } \\
\text { Cardiac Arrest } \\
\text { network: toward } \\
\text { Prevention, } \\
\text { Education and } \\
\text { NEw Treatment }\end{array}$ & $\begin{array}{l}\text { Disease cohorts. } \\
\text { Population } \\
\text { cohorts }\end{array}$ & $\begin{array}{l}\text { Czech Republic, } \\
\text { Denmark, France, } \\
\text { Italy, Sweden, The } \\
\text { Netherlands }\end{array}$ & $\begin{array}{l}\text { Aims to study: (1) risk factors and } \\
\text { mechanisms for the occurrence of } \\
\text { sudden cardiac arrest (SCA) in the } \\
\text { population, and (2) risk factors and } \\
\text { treatment strategies for survival after } \\
\text { SCA on a European scale }\end{array}$ & $\begin{array}{l}\text { No information } \\
\text { obtained }\end{array}$ & Yes & $\begin{array}{l}\text { No information } \\
\text { obtained }\end{array}$ & $\begin{array}{l}\text { No } \\
\text { information } \\
\text { obtained }\end{array}$ & $\begin{array}{l}\text { No } \\
\text { information } \\
\text { obtained }\end{array}$ & $\begin{array}{l}\text { Patients with sudden } \\
\text { cardiac arrest }\end{array}$ \\
\hline $\begin{array}{l}\text { After Breast } \\
\text { Cancer Pooling } \\
\text { Project }\end{array}$ & $\begin{array}{l}\text { Disease cohorts } \\
\text { (one is based on } \\
\text { the follow-up of a } \\
\text { randomized } \\
\text { clinical controlled } \\
\text { trial) }\end{array}$ & $\begin{array}{l}\text { China (Shanghai), } \\
\text { US }\end{array}$ & $\begin{array}{l}\text { Examine the role of physical activity, } \\
\text { adiposity, dietary factors, supplement } \\
\text { use, and quality of life in breast cancer } \\
\text { prognosis }\end{array}$ & 4 & Yes & 18.314 & $20-83$ & $\begin{array}{l}\text { No } \\
\text { information } \\
\text { obtained }\end{array}$ & $\begin{array}{l}\text { Breast cancer survivors } \\
\text { (women). Cancers were } \\
\text { diagnosed between } \\
1976 \text { and } 2006\end{array}$ \\
\hline
\end{tabular}


TABLE 1 | Continued

\begin{tabular}{|c|c|c|c|c|c|c|c|c|c|}
\hline Initiative & Types of cohorts & $\begin{array}{l}\text { Region, Country } \\
\text { were the cohorts } \\
\text { were collected }\end{array}$ & Main objective & $\begin{array}{l}\text { Number of } \\
\text { cohorts with } \\
\text { harmonized data }\end{array}$ & $\begin{array}{l}\text { More } \\
\text { cohorts } \\
\text { foreseen to } \\
\text { be } \\
\text { harmonized? }\end{array}$ & $\begin{array}{l}\text { Number of } \\
\text { participants } \\
\text { with } \\
\text { harmonized } \\
\text { data }\end{array}$ & $\begin{array}{l}\text { Age range } \\
\text { of the } \\
\text { sample }\end{array}$ & $\begin{array}{l}\text { No. of } \\
\text { harmonized } \\
\text { variables } \\
\text { (Maximum) }\end{array}$ & Population \\
\hline $\begin{array}{l}\text { B-CAST: Breast } \\
\text { CAncer } \\
\text { STratification }\end{array}$ & Disease cohorts & $\begin{array}{l}\text { No information } \\
\text { obtained }\end{array}$ & $\begin{array}{l}\text { In B-CAST tools will be developed to } \\
\text { allow precise identification of the } \\
\text { individual risk of breast cancer, the } \\
\text { subtype of cancer that is most likely to } \\
\text { develop and the prognosis of that } \\
\text { particular subtype }\end{array}$ & $\begin{array}{l}\text { No information } \\
\text { obtained }\end{array}$ & $\begin{array}{l}\text { No information } \\
\text { obtained }\end{array}$ & $\begin{array}{l}\text { No information } \\
\text { obtained }\end{array}$ & $\begin{array}{l}\text { No } \\
\text { information } \\
\text { obtained }\end{array}$ & $\begin{array}{l}\text { No } \\
\text { information } \\
\text { obtained }\end{array}$ & $\begin{array}{l}\text { Patients with breast } \\
\text { cancer }\end{array}$ \\
\hline $\begin{array}{l}\text { Collaborative } \\
\text { Group on } \\
\text { Epidemiological } \\
\text { Studies of Ovarian } \\
\text { Cancer }\end{array}$ & Disease cohorts & Worldwide & Study risk factors of oavarian cancer & 58 & $\begin{array}{l}\text { No information } \\
\text { obtained }\end{array}$ & 31,000 & $\begin{array}{l}\text { No } \\
\text { information } \\
\text { obtained }\end{array}$ & $\begin{array}{l}\text { No } \\
\text { information } \\
\text { obtained }\end{array}$ & $\begin{array}{l}\text { Women with ovarian } \\
\text { cancer }\end{array}$ \\
\hline $\begin{array}{l}\text { GENIE: Genomics } \\
\text { Evidence } \\
\text { Neoplasia } \\
\text { Information } \\
\text { Exchange }\end{array}$ & Disease cohorts & $\begin{array}{l}\text { Canada, France, } \\
\text { Netherlands, } \\
\text { Spain, UK, USA }\end{array}$ & $\begin{array}{l}\text { It is a multi-phase, multi-year, } \\
\text { international data-sharing project that } \\
\text { aims to catalyze precision cancer } \\
\text { medicine }\end{array}$ & 19 & Yes & 70,000 & All ages & $\begin{array}{l}\text { No } \\
\text { information } \\
\text { obtained }\end{array}$ & $\begin{array}{l}\text { Cancer patients treated } \\
\text { at multiple international } \\
\text { institutions }\end{array}$ \\
\hline $\begin{array}{l}\text { HARMONY: } \\
\text { European } \\
\text { Public-Private } \\
\text { Partnership for Big } \\
\text { Data in } \\
\text { Hematology }\end{array}$ & $\begin{array}{l}\text { Disease cohorts. } \\
\text { Clinical trials }\end{array}$ & All Europe & $\begin{array}{l}\text { The HARMONY Alliance uses big data } \\
\text { technologies to improve the treatment of } \\
\text { seven hematologic malignancies }\end{array}$ & $\begin{array}{l}\text { Acute Myeloid } \\
\text { Leukemia: } 5 \text { patient } \\
\text { cohorts. Multiple } \\
\text { myeloma: } 15 \\
\text { patient cohorts }\end{array}$ & In progress & $\begin{array}{l}11,664 \text { (aims } \\
\text { to harmonize } \\
\text { between } \\
75,000 \text { and } \\
100,000 \\
\text { anonymized } \\
\text { hematologic } \\
\text { patients by the } \\
\text { end of the } \\
\text { funding } \\
\text { period) }\end{array}$ & $\begin{array}{l}\text { All ages are } \\
\text { considered }\end{array}$ & $\begin{array}{l}\text { It depends on } \\
\text { the specific } \\
\text { research } \\
\text { question }\end{array}$ & $\begin{array}{l}\text { Patients with blood } \\
\text { malignancies }\end{array}$ \\
\hline $\begin{array}{l}\text { International } \\
\text { Collaboration of } \\
\text { Epidemiological } \\
\text { Studies of Cervical } \\
\text { Cancer }\end{array}$ & Disease cohorts & $\begin{array}{l}\text { Costa Rica, } \\
\text { Denmark, Norway, } \\
\text { Sweden, UK, US }\end{array}$ & $\begin{array}{l}\text { Study the effects of hormonal } \\
\text { contraceptive use and other factors on } \\
\text { the risk of cervical cancer }\end{array}$ & 9 & $\begin{array}{l}\text { No information } \\
\text { obtained }\end{array}$ & 2,109 & $\begin{array}{l}\text { No } \\
\text { information } \\
\text { obtained }\end{array}$ & $\begin{array}{l}\text { No } \\
\text { information } \\
\text { obtained }\end{array}$ & $\begin{array}{l}\text { Women with cervical } \\
\text { cancer }\end{array}$ \\
\hline $\begin{array}{l}\text { MaGIC: Malignant } \\
\text { Germ Cell } \\
\text { International } \\
\text { Consortium }\end{array}$ & Disease cohorts & $\begin{array}{l}\text { No information } \\
\text { obtained }\end{array}$ & $\begin{array}{l}\text { Developing more effective treatments for } \\
\text { germ cell tumors (GCT) through } \\
\text { scientific inquiry }\end{array}$ & $\begin{array}{l}\text { No information } \\
\text { obtained }\end{array}$ & $\begin{array}{l}\text { No information } \\
\text { obtained }\end{array}$ & $\begin{array}{l}\text { No information } \\
\text { obtained }\end{array}$ & $\begin{array}{l}\text { No } \\
\text { information } \\
\text { obtained }\end{array}$ & $\begin{array}{l}\text { No } \\
\text { information } \\
\text { obtained }\end{array}$ & $\begin{array}{l}\text { GCT patients all over } \\
\text { the world }\end{array}$ \\
\hline $\begin{array}{l}\mathrm{NCl} \text { : National } \\
\text { Cancer Institute } \\
\text { Cohort } \\
\text { Consortium }\end{array}$ & $\begin{array}{l}\text { Disease cohorts. } \\
\text { Population } \\
\text { cohorts. Clinical } \\
\text { trials }\end{array}$ & $\begin{array}{l}\text { Australia, Canada, } \\
\text { New Zealand, } \\
\text { USA }\end{array}$ & $\begin{array}{l}\text { Foster communication among } \\
\text { investigators leading cohort studies of } \\
\text { cancer, promote collaborative research } \\
\text { projects for topics not easily addressed } \\
\text { in a single study and identify common } \\
\text { challenges in cohort research and } \\
\text { search for solutions }\end{array}$ & $\begin{array}{l}\text { No information } \\
\text { obtained }\end{array}$ & Yes & $\begin{array}{l}\text { No information } \\
\text { obtained }\end{array}$ & $18+$ & $\begin{array}{l}\text { No } \\
\text { information } \\
\text { obtained }\end{array}$ & $\begin{array}{l}\text { Breast and colon family } \\
\text { cancer patients and } \\
\text { their families }\end{array}$ \\
\hline
\end{tabular}


TABLE 1 | Continued

\begin{tabular}{|c|c|c|c|c|c|c|c|c|c|}
\hline Initiative & Types of cohorts & $\begin{array}{l}\text { Region, Country } \\
\text { were the cohorts } \\
\text { were collected }\end{array}$ & Main objective & $\begin{array}{l}\text { Number of } \\
\text { cohorts with } \\
\text { harmonized data }\end{array}$ & $\begin{array}{l}\text { More } \\
\text { cohorts } \\
\text { foreseen to } \\
\text { be } \\
\text { harmonized? }\end{array}$ & $\begin{array}{l}\text { Number of } \\
\text { participants } \\
\text { with } \\
\text { harmonized } \\
\text { data }\end{array}$ & $\begin{array}{l}\text { Age range } \\
\text { of the } \\
\text { sample }\end{array}$ & $\begin{array}{l}\text { No. of } \\
\text { harmonized } \\
\text { variables } \\
\text { (Maximum) }\end{array}$ & Population \\
\hline $\begin{array}{l}\text { Second primary } \\
\text { malignancies in } \\
\text { thyroid cancer } \\
\text { patients }\end{array}$ & Disease cohorts & $\begin{array}{l}\text { France, Italy, } \\
\text { Sweden }\end{array}$ & $\begin{array}{l}\text { Evaluate the risk of second cancer and } \\
\text { leukemia in patients with papillary or } \\
\text { follicular thyroid cancer treated with } \\
\text { radioiodine or external beam radiation } \\
\text { therapy }\end{array}$ & 3 & No & 6,841 & $\begin{array}{l}7-80 \text { (at time } \\
\text { of diagnosis } \\
\text { of thyroid } \\
\text { cancer) }\end{array}$ & Around 10 & $\begin{array}{l}\text { Patients with papillary } \\
\text { or follicular thyroid } \\
\text { cancer }\end{array}$ \\
\hline $\begin{array}{l}\text { The International } \\
\text { CLL-IPI working } \\
\text { group }\end{array}$ & $\begin{array}{l}\text { Disease cohorts. } \\
\text { Clinical trials }\end{array}$ & $\begin{array}{l}\text { France, Germany, } \\
\text { Poland, UK, US }\end{array}$ & $\begin{array}{l}\text { We established an international } \\
\text { consortium with the aim to create an } \\
\text { international prognostic index for chronic } \\
\text { lymphocytic leukemia (CLL-IPI) that } \\
\text { integrates the major prognostic } \\
\text { parameters }\end{array}$ & 2 & & 1,254 & $\begin{array}{l}\text { No } \\
\text { information } \\
\text { obtained }\end{array}$ & 18 & $\begin{array}{l}\text { Chronic lymphocytic } \\
\text { leukemia patients }\end{array}$ \\
\hline $\begin{array}{l}\text { COASt: Clinical } \\
\text { Outcomes in } \\
\text { Arthroplasty Study }\end{array}$ & Disease cohorts & Europe & $\begin{array}{l}\text { Describe whether body mass index is a } \\
\text { clinically meaningful predictor of patient } \\
\text { reported outcomes following primary } \\
\text { total hip replacement (THR) surgery }\end{array}$ & 4 & $\begin{array}{l}\text { No information } \\
\text { obtained }\end{array}$ & 4,413 & $\begin{array}{l}\text { No } \\
\text { information } \\
\text { obtained }\end{array}$ & 24 & $\begin{array}{l}\text { Patients receiving } \\
\text { primary THR for } \\
\text { osteoarthritis }\end{array}$ \\
\hline $\begin{array}{l}\text { MARC-35: 35th } \\
\text { Multicenter Airway } \\
\text { Research } \\
\text { Collaboration }\end{array}$ & Disease cohorts & US & $\begin{array}{l}\text { Examine the association between the } \\
\text { infectious etiology of a child's severe } \\
\text { bronchiolitis and the level of serum } \\
25 \text {-hydroxyvitamin } \mathrm{D}(25[\mathrm{OH}] \mathrm{D}) \text { during } \\
\text { severe bronchiolitis, with the severity of } \\
\text { this illness, and the subsequent } \\
\text { development of recurrent wheezing by } \\
\text { age } 3 \text { years and combine these clinical } \\
\text { and laboratory data to derive the } \\
\text { wheezing index that will identify children } \\
\text { at higher risk of developing recurrent } \\
\text { wheezing by age } 3 \text { years }\end{array}$ & 17 & No & 920 & $0-1$ & Thousands & $\begin{array}{l}\text { Children age }<1 \text { year } \\
\text { hospitalized with severe } \\
\text { bronchiolitis }\end{array}$ \\
\hline $\begin{array}{l}\text { COSMIC: Cohort } \\
\text { Studies of Memory } \\
\text { in an International } \\
\text { Consortium }\end{array}$ & Disease cohorts & The world & $\begin{array}{l}\text { Harmonizing shared, non-identifiable } \\
\text { data from cohort studies that } \\
\text { longitudinally examine change in } \\
\text { cognitive function and the development } \\
\text { of dementia in older individuals ( } 60+ \\
\text { years). }\end{array}$ & $\begin{array}{l}\text { Data are } \\
\text { harmonized on a } \\
\text { project-by-project } \\
\text { basis, and only } \\
\text { subgroups of the } \\
\text { member studies } \\
\text { contribute to } \\
\text { particular projects }\end{array}$ & Yes & $\begin{array}{l}\text { Data are } \\
\text { harmonized } \\
\text { on a project- } \\
\text { by-project } \\
\text { basis, and } \\
\text { only } \\
\text { subgroups of } \\
\text { the member } \\
\text { studies } \\
\text { contribute to } \\
\text { particular } \\
\text { projects }\end{array}$ & 40-105 & $\begin{array}{l}\text { Harmonization } \\
\text { is done on a } \\
\text { project-by- } \\
\text { project basis } \\
\text { and the } \\
\text { number of } \\
\text { studies per } \\
\text { project } \\
\text { varies. For } \\
\text { the largest } \\
\text { project with } \\
20 \text { studies } \\
\text { there are } 16 \\
\text { harmonized } \\
\text { variables }\end{array}$ & $\begin{array}{l}60+\text { years old } \\
\text { individuals from } 29 \\
\text { countries all over the } \\
\text { world }\end{array}$ \\
\hline
\end{tabular}


TABLE 1 | Continued

\begin{tabular}{|c|c|c|c|c|c|c|c|c|c|}
\hline Initiative & Types of cohorts & $\begin{array}{l}\text { Region, Country } \\
\text { were the cohorts } \\
\text { were collected }\end{array}$ & Main objective & $\begin{array}{l}\text { Number of } \\
\text { cohorts with } \\
\text { harmonized data }\end{array}$ & $\begin{array}{l}\text { More } \\
\text { cohorts } \\
\text { foreseen to } \\
\text { be } \\
\text { harmonized? }\end{array}$ & $\begin{array}{l}\text { Number of } \\
\text { participants } \\
\text { with } \\
\text { harmonized } \\
\text { data }\end{array}$ & $\begin{array}{l}\text { Age range } \\
\text { of the } \\
\text { sample }\end{array}$ & $\begin{array}{l}\text { No. of } \\
\text { harmonized } \\
\text { variables } \\
\text { (Maximum) }\end{array}$ & Population \\
\hline $\begin{array}{l}\text { Lifebrain: Healthy } \\
\text { minds } 0-100 \\
\text { years: Optimizing } \\
\text { the use of } \\
\text { European brain } \\
\text { imaging cohorts }\end{array}$ & $\begin{array}{l}\text { Disease cohorts. } \\
\text { Population } \\
\text { cohorts }\end{array}$ & Western Europe & $\begin{array}{l}\text { Maximize the exploitation of brain } \\
\text { imaging cohorts by bringing together } \\
\text { studies on how differences and changes } \\
\text { in brain age relate to cognitive function } \\
\text { and mental health }\end{array}$ & $\begin{array}{l}1 \text { of anxiety and } \\
\text { depression patients }\end{array}$ & $\begin{array}{l}\text { No information } \\
\text { obtained }\end{array}$ & 2,981 & $\begin{array}{l}\text { No } \\
\text { information } \\
\text { obtained }\end{array}$ & $\begin{array}{l}\text { No } \\
\text { information } \\
\text { obtained }\end{array}$ & $\begin{array}{l}\text { Patients with anxiety or } \\
\text { depression }\end{array}$ \\
\hline $\begin{array}{l}\text { Seasonal plasticity } \\
\text { of cognition }\end{array}$ & $\begin{array}{l}\text { Disease cohorts. } \\
\text { Population } \\
\text { cohorts }\end{array}$ & $\begin{array}{l}\text { Canada, France, } \\
\text { US }\end{array}$ & $\begin{array}{l}\text { Test the hypotheses that season has a } \\
\text { significant association with cognition, } \\
\text { the odds of being diagnosed with mild } \\
\text { cognitive impairment or dementia, } \\
\text { cerebrospinal fluid Alzheimer disease } \\
\text { biomarkers, and the expression of } \\
\text { cognition-associated modules of } \\
\text { coexpressed genes in the human brain }\end{array}$ & 2 & $\begin{array}{l}\text { No information } \\
\text { obtained }\end{array}$ & 592 & $\begin{array}{l}\text { No } \\
\text { information } \\
\text { obtained }\end{array}$ & $\begin{array}{l}\text { No } \\
\text { information } \\
\text { obtained }\end{array}$ & $\begin{array}{l}\text { Alzheimer disease } \\
\text { patients or patients } \\
\text { with cognitive disorders } \\
\text { visited in tertiary care } \\
\text { clinics }\end{array}$ \\
\hline $\begin{array}{l}\text { HarmonicSS: } \\
\text { HARMONIzation } \\
\text { and integrative } \\
\text { analysis of } \\
\text { regional, national } \\
\text { and international } \\
\text { Cohorts on } \\
\text { primary Sjögren's } \\
\text { Syndrome (pSS) } \\
\text { toward improved } \\
\text { stratification, } \\
\text { treatment and } \\
\text { health policy } \\
\text { making disease }\end{array}$ & $\begin{array}{l}\text { Disease cohorts. } \\
\text { Clinical trials }\end{array}$ & Europe, US & $\begin{array}{l}\text { To bring together the largest } \\
\text { well-characterized regional, national and } \\
\text { international longitudinal cohorts of } \\
\text { patients with Primary Sjögren's } \\
\text { Syndrome (pSS) including those } \\
\text { participating in clinical trials, and by } \\
\text { taking into consideration the ethical, } \\
\text { legal, privacy and intelectual propiety } \\
\text { rights issues for sharing data from } \\
\text { different countries, to semantically } \\
\text { interlink and harmonize them into an } \\
\text { integrative pSS cohort structure on the } \\
\text { cloud }\end{array}$ & $\begin{array}{l}\text { No information } \\
\text { obtained }\end{array}$ & $\begin{array}{l}\text { No information } \\
\text { obtained }\end{array}$ & $\begin{array}{l}\text { No information } \\
\text { obtained }\end{array}$ & $\begin{array}{l}\text { No } \\
\text { information } \\
\text { obtained }\end{array}$ & $\begin{array}{l}\text { No } \\
\text { information } \\
\text { obtained }\end{array}$ & $\begin{array}{l}\text { Cohorts and clinical } \\
\text { trials of patients with } \\
\text { Primary Sjögren's } \\
\text { Syndrome }\end{array}$ \\
\hline $\begin{array}{l}\text { SABER: SAfety } \\
\text { Assessment of } \\
\text { Biologic ThERapy }\end{array}$ & Disease cohorts & US & $\begin{array}{l}\text { Understanding the absolute and } \\
\text { comparative risks of adverse events of } \\
\text { biologic treatments for patients with } \\
\text { autoimmune diseases }\end{array}$ & 4 & $\begin{array}{l}\text { No information } \\
\text { obtained }\end{array}$ & 239,806 & All ages & $\begin{array}{l}\text { No } \\
\text { information } \\
\text { obtained }\end{array}$ & $\begin{array}{l}\text { Patients with } \\
\text { autoimmune diseases } \\
\text { who had at least one } \\
\text { dispensing of a biologic } \\
\text { agent or comparison } \\
\text { non-biologic regimen } \\
\text { relevant to their } \\
\text { autoimmune disease }\end{array}$ \\
\hline $\begin{array}{l}\text { Thousand Faces } \\
\text { of Lupus }\end{array}$ & Disease cohorts & Canada & $\begin{array}{l}\text { Evaluate factors affecting therapeutic } \\
\text { approaches used in clinical practice for } \\
\text { the management of systemic lupus } \\
\text { erythematosus (SLE), in a multicenter } \\
\text { cohort }\end{array}$ & 10 & $\begin{array}{l}\text { No information } \\
\text { obtained }\end{array}$ & 1,497 & $\begin{array}{l}\text { No } \\
\text { information } \\
\text { obtained }\end{array}$ & $\begin{array}{l}\text { No } \\
\text { information } \\
\text { obtained }\end{array}$ & $\begin{array}{l}\text { Patients who meet } \\
\text { American College of } \\
\text { Rheumatology (ACR) } \\
\text { criteria for Systemic } \\
\text { Lupus Erythematosus }\end{array}$ \\
\hline
\end{tabular}


TABLE 1 | Continued

\begin{tabular}{|c|c|c|c|c|c|c|c|c|c|}
\hline Initiative & Types of cohorts & $\begin{array}{l}\text { Region, Country } \\
\text { were the cohorts } \\
\text { were collected }\end{array}$ & Main objective & $\begin{array}{l}\text { Number of } \\
\text { cohorts with } \\
\text { harmonized data }\end{array}$ & $\begin{array}{l}\text { More } \\
\text { cohorts } \\
\text { foreseen to } \\
\text { be } \\
\text { harmonized? }\end{array}$ & $\begin{array}{l}\text { Number of } \\
\text { participants } \\
\text { with } \\
\text { harmonized } \\
\text { data }\end{array}$ & $\begin{array}{l}\text { Age range } \\
\text { of the } \\
\text { sample }\end{array}$ & $\begin{array}{l}\text { No. of } \\
\text { harmonized } \\
\text { variables } \\
\text { (Maximum) }\end{array}$ & Population \\
\hline $\begin{array}{l}\text { Tumor Necrosis } \\
\text { Factor alpha } \\
\text { antagonist use } \\
\text { and cancer in } \\
\text { patients with } \\
\text { rheumatoid } \\
\text { arthritis }\end{array}$ & Disease cohorts & Canada and US & $\begin{array}{l}\text { Estimate the association between } \\
\text { treatment with biologic } \\
\text { disease-modifying antirheumatic drugs } \\
\text { (DMARDs) and development of cancer } \\
\text { in patients with rheumatoid arthritis }\end{array}$ & 3 & $\begin{array}{l}\text { No information } \\
\text { obtained }\end{array}$ & 8,458 & $65+$ & $\begin{array}{l}\text { No } \\
\text { information } \\
\text { obtained }\end{array}$ & $\begin{array}{l}\text { Rheumathoid arthritis } \\
\text { patients who had been } \\
\text { prescribed DMARDs or } \\
\text { methotrexate }\end{array}$ \\
\hline $\begin{array}{l}\text { GEMRIC: Global } \\
\text { ECT-MRI } \\
\text { Research } \\
\text { Collaboration }\end{array}$ & Disease cohorts & $\begin{array}{l}\text { Japan, Western } \\
\text { Europe and US. } \\
\text { Currently } \\
\text { approaching } \\
\text { China }\end{array}$ & $\begin{array}{l}\text { Creating a large database of multi-site } \\
\text { imaging data and } \\
\text { clinical/behavioral/physiological and } \\
\text { metadata for analysis of the neural } \\
\text { mechanisms and predictors of } \\
\text { electroconvulsive therapy-related clinical } \\
\text { response }\end{array}$ & 15 & Yes & 345 & $19-86$ & $\begin{array}{l}\text { More than a } \\
\text { thousand } \\
\text { because the } \\
\text { initiative } \\
\text { includes } \\
\text { diagnostic } \\
\text { imaging } \\
\text { variables }\end{array}$ & $\begin{array}{l}\text { Patients receiving } \\
\text { electroconvulsive } \\
\text { therapy }\end{array}$ \\
\hline $\begin{array}{l}\text { Predictors and } \\
\text { moderators of } \\
\text { cognitive and } \\
\text { behavioral therapy } \\
\text { outcomes for } \\
\text { obsessive- } \\
\text { compulsive } \\
\text { dissorder }\end{array}$ & $\begin{array}{l}\text { Disease cohorts. } \\
\text { Clinical trials }\end{array}$ & $\begin{array}{l}\text { Australia, Canada, } \\
\text { Europe, and US }\end{array}$ & $\begin{array}{l}\text { Identify potential factors that affect the } \\
\text { outcome of cognitive and behavioral } \\
\text { treatments of obsessive-compulsive } \\
\text { disorders }\end{array}$ & 8 & No & 359 & $\begin{array}{l}18+\text { (very few } \\
\text { over } 65)\end{array}$ & Around 20 & $\begin{array}{l}\text { Patients with } \\
\text { obsessive-compulsive } \\
\text { disorders }\end{array}$ \\
\hline $\begin{array}{l}\text { Antibiotic } \\
\text { treatment and } \\
\text { survival of nursing } \\
\text { home patients } \\
\text { with lower } \\
\text { respiratory tract } \\
\text { infection }\end{array}$ & Disease cohorts & $\begin{array}{l}\text { The Netherlands } \\
\text { and US }\end{array}$ & $\begin{array}{l}\text { Assess the effects of different antibiotic } \\
\text { treatment strategies on survival of elderly } \\
\text { nursing home residents with lower } \\
\text { respiratory tract infections in the } \\
\text { United States and the Netherlands, } \\
\text { where treatment approaches are quite } \\
\text { different }\end{array}$ & 2 & No & 1,221 & $70+$ & Around 40 & $\begin{array}{l}\text { Elderly nursing home } \\
\text { residents with lower } \\
\text { respiratory tract } \\
\text { infections }\end{array}$ \\
\hline $\begin{array}{l}\text { ART-CC: } \\
\text { Antiretroviral } \\
\text { Therapy Cohort } \\
\text { Collaboration }\end{array}$ & Disease cohorts & $\begin{array}{l}\text { Western Europe } \\
\text { and North America }\end{array}$ & $\begin{array}{l}\text { Estimate prognosis of HIV-1 positive, } \\
\text { treatment naiive patients initiating highly } \\
\text { active antiretroviral therapy (ART) }\end{array}$ & $\begin{array}{l}\text { No information } \\
\text { obtained }\end{array}$ & $\begin{array}{l}\text { No information } \\
\text { obtained }\end{array}$ & $\begin{array}{l}\text { No information } \\
\text { obtained }\end{array}$ & $\begin{array}{l}\text { No } \\
\text { information } \\
\text { obtained }\end{array}$ & $\begin{array}{l}\text { No } \\
\text { information } \\
\text { obtained }\end{array}$ & $\begin{array}{l}\text { HIV-1 positive, } \\
\text { treatment nailve } \\
\text { patients cohorts from } \\
\text { Europe and North } \\
\text { America }\end{array}$ \\
\hline
\end{tabular}


TABLE 1 | Continued

\begin{tabular}{|c|c|c|c|c|c|c|c|c|c|}
\hline Initiative & Types of cohorts & $\begin{array}{l}\text { Region, Country } \\
\text { were the cohorts } \\
\text { were collected }\end{array}$ & Main objective & $\begin{array}{l}\text { Number of } \\
\text { cohorts with } \\
\text { harmonized data }\end{array}$ & $\begin{array}{l}\text { More } \\
\text { cohorts } \\
\text { foreseen to } \\
\text { be } \\
\text { harmonized? }\end{array}$ & $\begin{array}{l}\text { Number of } \\
\text { participants } \\
\text { with } \\
\text { harmonized } \\
\text { data }\end{array}$ & $\begin{array}{l}\text { Age range } \\
\text { of the } \\
\text { sample }\end{array}$ & $\begin{array}{l}\text { No. of } \\
\text { harmonized } \\
\text { variables } \\
\text { (Maximum) }\end{array}$ & Population \\
\hline $\begin{array}{l}\text { COHERE: } \\
\text { Collaboration of } \\
\text { Observational HIV } \\
\text { Epidemiological } \\
\text { Research Europe }\end{array}$ & Disease cohorts & $\begin{array}{l}\text { Western Europe } \\
\text { and North America }\end{array}$ & $\begin{array}{l}\text { Pool and harmonize existing longitudinal } \\
\text { data on HIV-positive persons collected } \\
\text { across Europe to answer key research } \\
\text { questions that, in the era of potent } \\
\text { combination antiretroviral therapy } \\
\text { (cART), could not be addressed } \\
\text { adequately by individual cohorts }\end{array}$ & $\begin{array}{l}\text { No information } \\
\text { obtained }\end{array}$ & $\begin{array}{l}\text { No information } \\
\text { obtained }\end{array}$ & $\begin{array}{l}\text { No information } \\
\text { obtained }\end{array}$ & $\begin{array}{l}\text { No } \\
\text { information } \\
\text { obtained }\end{array}$ & Not reported & $\begin{array}{l}\text { HIV-infected people } \\
\text { residing in Europe }\end{array}$ \\
\hline $\begin{array}{l}\text { Early Antibiotic } \\
\text { Treatment for } \\
\text { Pediatric Febrile } \\
\text { Urinary Tract } \\
\text { Infection and } \\
\text { Renal Scarring }\end{array}$ & Disease cohorts & US & $\begin{array}{l}\text { Determine, in a well-characterized } \\
\text { sample of children with febrile urinary } \\
\text { track infections, whether delay in the } \\
\text { initiation of antimicrobial therapy was } \\
\text { associated with the occurrence and } \\
\text { severity of renal scarring and to } \\
\text { determine whether these associations } \\
\text { persisted after adjusting for potential } \\
\text { confounding factors }\end{array}$ & 2 & No & 802 & 2-72 months & $\begin{array}{l}\text { No } \\
\text { information } \\
\text { obtained }\end{array}$ & $\begin{array}{l}\text { Children aged 2-72 } \\
\text { months with a urinary } \\
\text { tract infection } \\
\text { producing fever }\end{array}$ \\
\hline $\begin{array}{l}\text { HAART and early } \\
\text { mortality }\end{array}$ & Disease cohorts & Brazil and US & $\begin{array}{l}\text { Compare the early mortality pattern and } \\
\text { the causes of death among patients } \\
\text { starting HAART in Brazil and the } \\
\text { United States }\end{array}$ & 2 & $\begin{array}{l}\text { No information } \\
\text { obtained }\end{array}$ & 1,774 & $\begin{array}{l}\text { No } \\
\text { information } \\
\text { obtained }\end{array}$ & 10 & HIV-infected patients \\
\hline $\begin{array}{l}\text { leDEA: } \\
\text { International } \\
\text { epidemiology } \\
\text { Databases to } \\
\text { Evaluate AIDS }\end{array}$ & Disease cohorts & $\begin{array}{l}\text { Africa, Asia-Pacific } \\
\text { region, the } \\
\text { Central/South } \\
\text { America/Caribbean } \\
\text { region, and North } \\
\text { America }\end{array}$ & $\begin{array}{l}\text { Collect and define key variables, } \\
\text { harmonize data, and implement } \\
\text { methodology to effectively pool data as } \\
\text { a cost-effective means of generating } \\
\text { large data sets to address the high } \\
\text { priority research questions and } \\
\text { streamline HIV/AIDS research }\end{array}$ & $\begin{array}{l}\text { No information } \\
\text { obtained }\end{array}$ & $\begin{array}{l}\text { No information } \\
\text { obtained }\end{array}$ & $\begin{array}{l}\text { No information } \\
\text { obtained }\end{array}$ & $\begin{array}{l}\text { No } \\
\text { information } \\
\text { obtained }\end{array}$ & $\begin{array}{l}\text { No } \\
\text { information } \\
\text { obtained }\end{array}$ & $\begin{array}{l}\text { HIV/AIDS patients from } \\
\text { Africa, the Asia-Pacific } \\
\text { region, the } \\
\text { Central/South } \\
\text { America/Caribbean } \\
\text { region, and North } \\
\text { America }\end{array}$ \\
\hline $\begin{array}{l}\text { ReCoDID: } \\
\text { Reconciliation of } \\
\text { Cohort data in } \\
\text { Infectious } \\
\text { Diseases }\end{array}$ & Disease cohorts & $\begin{array}{l}\text { No information } \\
\text { obtained }\end{array}$ & $\begin{array}{l}\text { Develop an equitable, accessible, and } \\
\text { sustainable model for the storage, } \\
\text { curation, and analyses of } \\
\text { clinical-epidemiological and } \\
\text { high-dimensional sample data collected } \\
\text { by infectious disease cohorts in } \\
\text { low-and-midle-income countries }\end{array}$ & $\begin{array}{l}\text { No information } \\
\text { obtained }\end{array}$ & In progress & $\begin{array}{l}\text { No information } \\
\text { obtained }\end{array}$ & $\begin{array}{l}\text { No } \\
\text { information } \\
\text { obtained }\end{array}$ & $\begin{array}{l}\text { No } \\
\text { information } \\
\text { obtained }\end{array}$ & $\begin{array}{l}\text { Patients with infectious } \\
\text { diseases }\end{array}$ \\
\hline $\begin{array}{l}\text { RESPOND: } \\
\text { International } \\
\text { Cohort } \\
\text { Consortium of } \\
\text { Infectious Disease }\end{array}$ & Disease cohorts & $\begin{array}{l}\text { Australia, Georgia } \\
\text { and Western } \\
\text { Europe }\end{array}$ & $\begin{array}{l}\text { Build an innovative, flexible and dynamic } \\
\text { cohort consortium for the study of } \\
\text { infectious diseases, including HIV and } \\
\text { people at risk for HIV, as a generic } \\
\text { structure for facilitating multi stakeholder } \\
\text { involvement }\end{array}$ & $\begin{array}{l}\text { No information } \\
\text { obtained }\end{array}$ & $\begin{array}{l}\text { No information } \\
\text { obtained }\end{array}$ & $\begin{array}{l}\text { No information } \\
\text { obtained }\end{array}$ & $\begin{array}{l}\text { No } \\
\text { information } \\
\text { obtained }\end{array}$ & $\begin{array}{l}\text { No } \\
\text { information } \\
\text { obtained }\end{array}$ & $\begin{array}{l}\text { People } 18+\text { at high risk } \\
\text { of acquiring HIV and } \\
\text { people living with HIV } \\
\text { and/or with other } \\
\text { infectious diseases or } \\
\text { across Europe, South } \\
\text { America and Australia }\end{array}$ \\
\hline
\end{tabular}


TABLE 1 | Continued

\begin{tabular}{|c|c|c|c|c|c|c|c|c|c|}
\hline Initiative & Types of cohorts & $\begin{array}{l}\text { Region, Country } \\
\text { were the cohorts } \\
\text { were collected }\end{array}$ & Main objective & $\begin{array}{l}\text { Number of } \\
\text { cohorts with } \\
\text { harmonized data }\end{array}$ & $\begin{array}{l}\text { More } \\
\text { cohorts } \\
\text { foreseen to } \\
\text { be } \\
\text { harmonized? }\end{array}$ & $\begin{array}{l}\text { Number of } \\
\text { participants } \\
\text { with } \\
\text { harmonized } \\
\text { data }\end{array}$ & $\begin{array}{l}\text { Age range } \\
\text { of the } \\
\text { sample }\end{array}$ & $\begin{array}{l}\text { No. of } \\
\text { harmonized } \\
\text { variables } \\
\text { (Maximum) }\end{array}$ & Population \\
\hline $\begin{array}{l}\text { Adults Born } \\
\text { Preterm } \\
\text { International } \\
\text { Collaboration }\end{array}$ & Disease cohorts & $\begin{array}{l}\text { Australia, Canada, } \\
\text { Finland, } \\
\text { Netherlands, } \\
\text { Northern Ireland, } \\
\text { Norway, US }\end{array}$ & $\begin{array}{l}\text { Our main aim was to identify factors that } \\
\text { either increase or decrease risk of high } \\
\text { blood pressure among adults born with } \\
\text { very low birth weight }\end{array}$ & 9 & $\begin{array}{l}\text { No information } \\
\text { obtained }\end{array}$ & $\begin{array}{l}1,571 \text { patients } \\
\text { and } 777 \\
\text { controls }\end{array}$ & $\begin{array}{l}\text { No } \\
\text { information } \\
\text { obtained }\end{array}$ & $\begin{array}{l}\text { No } \\
\text { information } \\
\text { obtained }\end{array}$ & $\begin{array}{l}\text { Very low birth weight } \\
\text { and very preterm } \\
\text { babies who reach } \\
\text { adulthood }\end{array}$ \\
\hline $\begin{array}{l}\text { Necrotizing } \\
\text { enterocolitis (NEC) } \\
\text { study }\end{array}$ & Disease cohorts & $\begin{array}{l}\text { Austria and The } \\
\text { Netherlands }\end{array}$ & $\begin{array}{l}\text { The first aim of the study was to } \\
\text { correlate the occurrence of a blood } \\
\text { stream infection (BSI) during the early } \\
\text { phase of necrotizing enterocolitis (NEC) } \\
\text { with intestinal fatty acid-binding protein } \\
\text { (I-FABP) levels, as a marker for loss of } \\
\text { gut wall integrity owing to mucosal } \\
\text { damage, and Interleukin (IL)-8 levels, as } \\
\text { a biomarker for the pro-inflammatory } \\
\text { cascade in NEC. The second aim of the } \\
\text { study was to investigate the relation } \\
\text { between the occurrence of a BSI and } \\
\text { disease outcome }\end{array}$ & 2 & $\begin{array}{l}\text { No information } \\
\text { obtained }\end{array}$ & 57 & 24-40 weeks & 13 & $\begin{array}{l}\text { Patients with } \\
\text { necrotizing enterocolitis }\end{array}$ \\
\hline $\begin{array}{l}\text { Recurrent leg } \\
\text { venous ulcers } \\
\text { study }\end{array}$ & Disease cohorts & Eastern Australia & $\begin{array}{l}\text { Identify risk and protective factors for } \\
\text { recurrence of venous leg ulcers }\end{array}$ & 3 & Yes & 250 & $26-96$ & 24 & $\begin{array}{l}\text { Patients with a healed } \\
\text { leg ulcer of primarily } \\
\text { venous etiology }\end{array}$ \\
\hline $\begin{array}{l}\text { MARS: Multicenter } \\
\text { AVM Research } \\
\text { Study }\end{array}$ & Disease cohorts & Scotland and US & $\begin{array}{l}\text { Identify risk factors for intracranial } \\
\text { hemorrhage in the natural history course } \\
\text { of brain arteriovenous malformations }\end{array}$ & 4 & Yes & 2,525 & $\begin{array}{l}\text { No } \\
\text { information } \\
\text { obtained }\end{array}$ & 13 & $\begin{array}{l}\text { Patients with } \\
\text { arteriovenous } \\
\text { malformations }\end{array}$ \\
\hline $\begin{array}{l}\text { Pulmonary } \\
\text { embolism } \\
\text { presentation }\end{array}$ & $\begin{array}{l}\text { Disease cohorts } \\
\text { (one clinical trial) }\end{array}$ & $\begin{array}{l}\text { Belgium, France } \\
\text { and Switzerland }\end{array}$ & $\begin{array}{l}\text { Compare clinical characteristics } \\
\text { between women and men with } \\
\text { suspected and confirmed pulmonary } \\
\text { embolism }(\mathrm{PE}) \text { and their impact on } \\
\text { clinical probability prediction scores and } \\
\text { on diagnostic work-up of } \mathrm{PE} \text {, and to } \\
\text { assess whether differences at } \\
\text { presentation could account for the } \\
\text { increased recurrence rate in men }\end{array}$ & 3 & No & 3,414 & $18-98$ & Around 30 & $\begin{array}{l}\text { Patients with a clinical } \\
\text { suspicion of pulmonary } \\
\text { embolism }\end{array}$ \\
\hline $\begin{array}{l}\text { BIOMAP: } \\
\text { Biomarkers in } \\
\text { Atopic Dermatitis } \\
\text { and Psoriasis }\end{array}$ & $\begin{array}{l}\text { Disease cohorts. } \\
\text { Clinical trials }\end{array}$ & $\begin{array}{l}\text { No information } \\
\text { obtained }\end{array}$ & $\begin{array}{l}\text { Examine the causes and mechanisms of } \\
\text { atopic dermatitis and psoriasis to enable } \\
\text { optimal treatments and an individualized } \\
\text { therapy scheme for each patient }\end{array}$ & $\begin{array}{l}\text { No information } \\
\text { obtained }\end{array}$ & In progress & $\begin{array}{l}\text { No information } \\
\text { obtained }\end{array}$ & $\begin{array}{l}\text { No } \\
\text { information } \\
\text { obtained }\end{array}$ & $\begin{array}{l}\text { No } \\
\text { information } \\
\text { obtained }\end{array}$ & $\begin{array}{l}\text { Patients with atopic } \\
\text { dermatitis and psoriasis }\end{array}$ \\
\hline
\end{tabular}

Recurrent leg
venous ulcers

study

AVM Research

embolism

Disease cohorts

Belgium, France

(

presentation could account for the

therapy scheme for each patient 
only include cohorts from high-income countries, and none harmonize data from LMIC countries alone.

Most initiatives are partnered with universities, hospitals, and research institutes. Governmental institutions take part in a few of them (9). The presence of patient associations and pharmaceutical companies as partners is anecdotal. The number of partners ranges between 2 and more than 100, with a median of 12 . Three quarters comprise 20 partners or fewer.

Most initiatives have been or are funded by American (12) or European (10) institutions. Canadian funding comes third (4). The vast majority have received public funding alone (22). Five have received combined funding from public institutions and non-profit organizations. Private funding was provided in isolation to one initiative (RESPOND), combined with public funding to another one (EHDEN), and combined with non-profit funding to a third one (Tumor necrosis factor $\alpha$ antagonist use).

Their objectives may be classified into four general categories (some initiatives share more than one): determining the prognosis of subgroups of patients (14), providing a repository of patients (11), establishing the efficacy (6) or safety (4) of treatments, and exploring risk factors and biomarkers of diseases (10).

The median number of cohorts included in each initiative is 5 , ranging from 1 (which also harmonizes population cohorts) to 58 ; three quarters include 17 cohorts or fewer. The number of individuals included varies wildly, from 57 to 310 million (Sentinel initiative). The median is 6,841. Eight out of 37 (21.6\%) initiatives have harmonized fewer than 1,000 patients and the same proportion have harmonized 100,000 patients or more. Twenty-six have harmonized all or almost all the cohorts incorporated to the initiative, two (EHDEN and CINECA) are still in the process of harmonizing their cohorts and another two (CNODES and COSMIC) harmonize data on a project-byproject basis.

Eight initiatives included patients from all ages, eight included only adult patients, three included only children, and two included exclusively older people.

\section{REFERENCES}

1. Blettner M, Sauerbrei W, Schlehofer B, Scheuchenpflug T, Friedenreich C. Traditional reviews, meta-analyses and pooled analyses in epidemiology. Int $J$ Epidemiol. (1999) 28:1-9. doi: 10.1093/ije/ 28.1.1

2. Hamilton CM, Strader LC, Pratt JG, Maiese D, Hendershot T, Kwok RK, et al. The PhenX toolkit: get the most from your measures. Am J Epidemiol. (2011) 174:253-60. doi: 10.1093/aje/k wr193

3. Ioannidis JPA, Rosenberg PS, Goedert JJ, O’Brien TR. Commentary: meta-analysis of individual participants' data in genetic epidemiology. Am J Epidemiol. (2002) 156:204-10. doi: 10.1093/aje/k wf031

4. Lyman GH, Kuderer NM. The strengths and limitations of metaanalyses based on aggregate data. BMC Med Res Methodol. (2005) 5:14. doi: $10.1186 / 1471-2288-5-14$
Of those which have declared the number of variables in their harmonized database, there are between 10 and more than 1,000 (median 24), with two out of 15 (13.3\%) including more than 1,000 variables.

Four initiatives harmonized administrative databases. Thirtythree were retrospective vs. four prospective. The great majority do not report major threats to the representativity of their samples.

\section{DATA AVAILABILITY STATEMENT}

The dataset generated by this study can be found in the webpage of the SYNCHROS project https://www.synchros.es.

\section{AUTHOR CONTRIBUTIONS}

ÁR-L performed the database searches, extracted the information from the initiatives, and drafted the article. ÁR-L, LR-U, and $\mathrm{CK}$ evaluated the initiatives against inclusion and exclusion criteria. All authors made substantial contributions to the conception and design of the paper, analysis and interpretation of data, reviewed the manuscript, and read and approved its final version.

\section{FUNDING}

This work was supported by the European Union's H2020 Programme under grant agreement number 825884.

\section{ACKNOWLEDGMENTS}

The authors want to express their gratitude to all principal investigators and project managers who answered our request for further information on their harmonization initiatives.

5. Thompson A. Thinking big: large-scale collaborative research in observational epidemiology. Eur J Epidemiol. (2009) 24:72731. doi: 10.1007/s10654-009-9412-1

6. World Health Organization. The Global Burden of Disease: 2004 Update. Geneva, Switzerland: World Health Organization (2004).

Conflict of Interest: The authors declare that the research was conducted in the absence of any commercial or financial relationships that could be construed as a potential conflict of interest.

Copyright @ 2021 Rodríguez-Laso, Rico-Uribe, Kubiak, Haro, Rodríguez-Mañas and Ayuso. This is an open-access article distributed under the terms of the Creative Commons Attribution License (CC BY). The use, distribution or reproduction in other forums is permitted, provided the original author(s) and the copyright owner(s) are credited and that the original publication in this journal is cited, in accordance with accepted academic practice. No use, distribution or reproduction is permitted which does not comply with these terms. 